\title{
AGREGAÇÃO DE FATORES DE RISCO CARDIOVASCULAR E OCORRÊNCIA DE HIPERTENSÃO ARTERIAL EM ADULTOS SEDENTÁRIOS
}

\author{
CLUSTER OF CARDIOVASCULAR RISK FACTORS AND OCCURRENCE OF ARTERIAL \\ HYPERTENSION IN SEDENTARY ADULTS
}

\author{
ACUMULACIÓN DE FACTORES DE RIESGO CARDIOVASCULAR Y OCURRENCIA \\ DE HIPERTENSIÓN ARTERIAL EN ADULTOS SEDENTARIOS
}

\begin{abstract}
Ferdinando Oliveira Carvalho (Educador Físico) $^{1}$

Rômulo Araújo Fernandes

(Educador Físico) $^{2}$

Diego Giulliano Destro Christofaro

(Educador Físico) $^{2}$

Jamile Sanches Codogno

(Educador Físico) ${ }^{2}$

Henrique Luiz Monteiro

(Educador Físico) $^{3}$

Sérgio Rodrigues Moreira

(Educador Físico) $^{4}$

Edilson Serpeloni Cyrino

(Educador Físico) $^{5}$

Carmen Silvia Grubert Campbell

(Educador Físico) $^{6}$

Herbert Gustavo Simões

(Educador Físico) $^{6}$

1. Programa de Pós-Graduação em Educação Física. Universidade Católica de Brasília - UCB -

Brasília, DF, Brasil; Universidade

Federal do Vale do São Francisco

- UNIVASF - Petrolina, PE, Brasil;

Grupo de Estudo e Pesquisa em

Metabolismo Nutrição e Exercício

- GEPEMENE.

2. Departamento de Educação

Física. Universidade Estadual

Paulista - UNESP - Presidente

Prudente, SP, Brasil.

3. Departamento de Educação

Física. Universidade Estadual

Paulista - UNESP - Bauru, SP, Brasil.

4. UNIVASF - Petrolina, PE, Brasil.

5. Departamento de Educação

Física. UEL - Londrina, PR, Brasil.

6. Programa de Pós-Graduação

em Educação Física. Universidade

Católica de Brasília - UCB -

Brasília, DF, Brasil.
\end{abstract}

\section{Correspondência:}

Diego Giulliano Destroy Christo

Faro - Departamento de Educação

Física - Universidade Estadual

Paulista - UNESP. Rua Roberto

Simonsen, 305, 19060-900,

Presidente Prudente, SP, Brasil.

diegochristofaro@yahoo.com.br

\section{RESUMO}

Objetivo: Analisar em uma grande amostra de adultos sedentários a possível associação entre hipertensão arterial e baixa aptidão cardiorrespiratória, obesidade geral e central. Métodos: A amostra foi composta por 1.092 adultos sedentários brasileiros (429 homens e 633 mulheres). Pressão arterial foi acessada e hipertensão arterial diagnosticada. Três fatores de risco cardiovascular foram considerados: obesidade geral (índice de massa corporal), obesidade abdominal (circunferência de cintura) e baixa aptidão cardiorrespiratória (teste submáximo em cicloergômetro). Resultados: A taxa de hipertensão arterial foi de 9,3\% (IC95\% = 7,6 - 11,2). Houve associação entre mais alta prevalência de hipertensão arterial e diagnóstico de obesidade $(p=0,001)$, valores elevados de circunferência de cintura $(p=0,001)$ e baixo $\operatorname{VO}_{2 \operatorname{máx}}(p=0,013)$. Independentemente de idade e sexo, indivíduos sedentários com simultaneamente baixo consumo máximo de oxigênio e obesidade geral $(\mathrm{RP}=5,21[\mathrm{IC} 95 \%=8,94-3,03] ; \mathrm{p}=0,001)$ ou abdominal $(\mathrm{RP}=4,05[\mathrm{IC}$ C5\% $=2,22-7,40] ; \mathrm{p}=0,001)$ apresentaram maior probabilidade de ser hipertenso que os sedentários com nenhum fator de risco. Conclusão: Adultos sedentários, independentemente de sexo e idade, a presença de baixa aptidão cardiorrespiratória está associada à hipertensão arterial apenas quando também há obesidade geral ou abdominal, indicando que a interação destas variáveis tem relevante peso na gênese da doença.

Palavras-chave: fatores de risco, aptidão física, obesidade, adulto.

\begin{abstract}
Objective: To analyze in a large sample of sedentary adults the possible association between arterial hypertension and decreased physical fitness, and increased general and central obesity. Methods: The sample was composed by 1,092 (429 male and 663 female) Brazilian sedentary adults. Blood pressure was assessed and arterial hypertension was diagnosed. Three cardiovascular risk factors were considered: general obesity (body mass index), central obesity (waist circumference) and decreased physical fitness (submaximal bicycle ergometer test). Results: Arterial hypertension rate was 9.3\% (95\% Cl $=7.6-11.2)$. There was association between higher occurrence of arterial hypertension and the diagnosis of obesity $(p=0.001)$, elevated values of WC $(p=0.001)$ and reduced values of $\mathrm{VO}_{2 \max }(p=0.013)$. Independently of gender and age, sedentary individuals with simultaneous decreased physical fitness plus general $(P R=5.21[95 \% \mathrm{Cl}=8.94-3.03] ; p=0.001)$ and central $(P R=4.05[95 \% \mathrm{Cl}=$ 2.22-7.40]; $p=0.001$ ) obesity presented higher likelihood to have arterial hypertension than sedentary individuals with none cardiovascular risk factor. Conclusion: Sedentary adults, independently of both gender and age, the presence of low cardiorespiratory fitness is associated with increased occurrence of arterial hypertension only when there is either increased central or general adiposity indicating that the interaction of these variables have relevant burden in the genesis of the disease.
\end{abstract}

Keywords: risk factors, physical fitness, obesity, adult.

\section{RESUMEN}

Objetivo: Analizar, en una muestra grande de adultos sedentarios, la posible vinculación entre hipertensión arterial y reducción de aptitud cardiorrespiratoria, con más obesidad general y central. Métodos: La muestra se compuso de 1.092 adultos sedentarios brasileños (429 hombres y 663 mujeres). La presión arterial fue evaluada y la hipertensión arterial fue diagnosticada. Tres factores de riesgo cardiovascular fueron considerados: obesidad general (índice de masa corporal), obesidad central (circunferencia de la cintura) y baja aptitud cardiorrespiratoria (prueba submáxima de ergometría en bicicleta). Resultados: La tasa de hipertensión arterial fue 9,3\% (IC95\% =7,6-11,2). Hubo asociación entre la prevalencia más alta de hipertensión arterial y el diagnóstico de obesidad $(p=0,001)$, valores altos de circunferencia de cintura $(p=0,001)$ y bajo VO $\operatorname{Vmax}_{\max }(p=0,013)$. Independientemente de edad y sexo, los individuos sedentarios con, simultáneamente, baja aptitud cardiorrespiratoria, y obesidad general 
$(R P=5,21$ [IC95\% $=8,94-3,03] ; p=0,001)$ y central $(R P=4,05$ [IC95\% $=2,22-7,40] ; p=0,001)$ presentaron más probabilidades de ser hipertensos que los sedentarios con ningún factor de riesgo cardiovascular. Conclusión: En adultos sedentarios, sin considerar sexo ni edad, la presencia de baja aptitud cardiorrespiratoria está vinculada a la hipertensión arterial solamente cuando hay adiposidad general o central, indicando que la interacción de estas variables tiene peso relevante en la génesis de la enfermedad.

Palabras clave: factores de riesgo, aptitud fisica, obesidad, adulto.

Artigo recebido em 29/04/2012, aprovado em 04/10/2013.

\section{INTRODUÇÃO}

Evidências têm oferecido suporte para a hipótese de que inatividade física está relacionada com maior produção de espécies reativas de oxigênio', lipoproteínas de baixa densidade oxidadas ${ }^{2}$ e, consequentemente, desenvolvimento de disfunção endotelial, levando a doenças cardiovasculares ${ }^{3,4}$. Tem sido demonstrado que a obesidade (principalmente a abdominal) tem papel central no desenvolvimento de doenças metabólicas e cardiovasculares, incluindo diabetes mellitus tipo 2 e hipertensão arterial ${ }^{3-5}$.

Hipertensão arterial constitui um desfecho cardiovascular de alta ocorrência em países desenvolvidos ${ }^{6}$ e em desenvolvimento ${ }^{7,8}$, a qual tem sido associada ao aumento de gastos públicos com saúde ${ }^{9}$. Maior nível de atividade física é consistentemente relacionado com maior aptidão cardiorrespiratória ${ }^{10} \mathrm{e}$ ambas são inversamente relacionadas à obesidade geral e abdomina $\left.\right|^{8,11}$. Assim, o efeito benéfico da aptidão cardiorrespiratória na prevenção/tratamento da hipertensão arterial são comumente atribuídos por modificações na composição corporal e prevenção do ganho de peso ${ }^{8}$. Contudo, não é totalmente claro o real peso da aptidão cardiorrespiratória e distribuição da adiposidade corporal na gênesis da hipertensão arterial.

Assim, o objetivo do presente estudo foi analisar, em uma grande amostra de adultos sedentários, a possível associação entre hipertensão arterial, baixa aptidão cardiorrespiratória e obesidade geral e abdominal.

\section{MÉTODOS}

\section{Amostra}

A amostra foi composta por 1.092 (429 homens e 663 mulheres) adultos sedentários (nenhuma atividade física sistematizada nos últimos seis meses prévios à avaliação do estudo [International Physical Activity Questionnaire - IPAQ]) da cidade de Londrina, Norte do Paraná. Estes adultos foram recrutados em uma grande academia da cidade e os dados apresentados foram coletados antes de iniciarem seu programa de exercícios. O estudo foi conduzido durante o ano de 2002. Todos os sujeitos envolvidos na amostra assinaram um Termo de Consentimento Livre e Esclarecido e o estudo foi previamente aprovado pelo Comitê de Ética da Universidade Federal do Vale do São Francisco - UNIVASF.

\section{Valores de pressão arterial}

Seguindo os procedimentos descritos por Perloff et al..$^{22}$, um aparelho auscultatório (esfigmomanômetro) foi usado para mensurar a pressão arterial sistólica (PAS), diastólica (PAD) e média (PAM). Anteriormente às medidas, um período de 10 minutos sentado em repouso foi respeitado. Todas as medidas foram feitas por um pesquisador treinado no braço esquerdo do avaliado e os sujeitos receberam orientações a serem seguidas antes das avaliações: 1) nenhum exercício vigoroso nas últimas 24 h; 2) nenhum consumo de álcool e cafeína nas últimas 24 h; 3) ao menos seis horas de sono na noite anterior. Hipertensão arterial foi definida de acordo com a Diretriz da Sociedade Interamericana de Hipertensão (PAS $\geq 140 \mathrm{mmHg}$ e/ou PAD $\geq 90 \mathrm{mmHg}$ em repouso).

\section{Fatores de risco cardiovascular}

Três fatores de risco cardiovascular foram considerados em nosso estudo: obesidade geral, obesidade abdominal e baixa aptidão cardiorrespiratória. Massa corporal $(\mathrm{kg})$ e estatura $(\mathrm{cm})$ foram mensuradas em uma balança digital e um estadiômetro de madeira ${ }^{13}$, respectivamente. O índice de massa corporal (IMC) foi calculado $\left(\mathrm{kg} / \mathrm{m}^{2}\right)$. Circunferência de cintura (CC) foi mensurada com uma fita metálica $(\mathrm{cm})$ no ponto médio entre a crista ilíaca e a última costela ${ }^{14}$. Valores de $I M C \geq 30 \mathrm{~kg} / \mathrm{m}^{2}$ foram usados para indicar obesidade geral e valores de $C C \geq 88 \mathrm{~cm}$ e $\geq 102 \mathrm{~cm}$ foram usados como indicadores de obesidade abdominal em mulheres e homens ${ }^{15}$, respectivamente.

Aptidão cardiorrespiratória foi expressa como consumo máximo de oxigênio $\left(\mathrm{VO}_{2 m a ́ x}[\mathrm{~mL} / \mathrm{kg} / \mathrm{min}]\right)$ e foi mensurada por meio de teste submáximo em ciclo ergômetro (modelo Movement Biocycle Electromagnetic), o qual foi conduzido de acordo com Astrand e Rodahl16. Valores de $\mathrm{VO}_{2 \text { máx }}$ foram estratificados em quartil de acordo com o sexo e, então, os sujeitos situados no mais baixo quartil (P25) compuseram o grupo de baixa aptidão cardiorrespiratória e consequentemente de mais alto risco cardiovascular.

Finalmente, uma variável categórica foi criada, na qual o número de fatores de risco cardiovascular alterados foi computado. Esta variável categórica variou de 0 (nenhum fator de risco alterado) a 3 (diagnóstico positivo de obesidade geral, central e baixa aptidão cardiorrespiratória).

\section{Procedimentos estatísticos}

Variáveis numéricas foram apresentadas como média e desvio padrão, ao passo que as categóricas foram apresentadas como taxas. O teste $t$ de Student para amostras independentes e o teste do Qui-quadrado compararam variáveis numéricas e categóricas, respectivamente. Análise de variância (ANOVA one way) e o post hoc de Tukey foram usados. Criou-se um modelo multivariado para dados categóricos (regressão de Poisson com ajuste robusto de variância), o qual foi expresso em valores de razão de prevalência (RP) e sues intervalos de confiança de $95 \%$. Ajustes neste modelo foram feitos pela inserção das variáveis: sexo e idade.

Significância estatística ( $p$ ) foi pré-fixada em valores de $p<0,05$ e o software utilizado foi o STATA (versão 8.0).

\section{RESULTADOS}

Na amostra analisada, a média de idade foi 27,6 anos e não houve diferença entre os sexos $(p=0,602)$. Por outro lado, homens apresentaram maiores valores de IMC, CC, PAS, PAD e PAM. Houve similaridade nos valores de $\mathrm{VO}_{2 \text { máx }}$ entre os sexos (tabela 1). Na amostra total, a idade foi positivamente relacionada à pressão arterial (PAS $r=0,18 \mathrm{e}$ PAD $r=0,17)$ e o mesmo padrão de correlação foi identificado para o IMC (PAS $r=0,51$ e PAD $r=0,46)$ e CC (PAS $r=0,52$ e PAD $r=0,49)$. valores de $\mathrm{VO}_{2 \text { máx }}$ foram inversamente relacionados à $\mathrm{PAS}(r=-0,16)$ e PAD $(r=-0,17)$.

A prevalência de hipertensão arterial foi de 9,3\% (IC95\% = 7,6 11,2). A obesidade geral e a abdominal foram detectadas em 4,3\% 
$(\mathrm{IC} 95 \%=3,1-5,6)$ e 5,4\% $(95 \% \mathrm{Cl}=4,2-7,1)$ da amostra, respectivamente. Houve associação entre maior ocorrência de hipertensão arterial e diagnóstico de obesidade (Qui-quadrado com $p=0,001$ ), valores elevados de CC (Qui-quadrado com $p=0,001$ ) e valores reduzidos de $\mathrm{VO}_{2 \text { máx }}$ (Qui-quadrado com $\mathrm{p}=0,013$ ) (tabela 2).

A amostra foi estratificada de acordo com o número de fatores de risco cardiovascular e foram observados maiores valores de PAS (ANOVA one way com $p=0,001$ ), PAD (ANOVA one way com $p=0,001$ ) e PAM (ANOVA one way com $p=0,001$ ) em sujeitos com presença simultânea de baixa aptidão cardiorrespiratória, obesidade geral e abdominal (figura 1).

Os grupos "obesidade geral" ( $p=0,001)$, obesidade geral mais obesidade abdominal ( $p=0,006)$, obesidade geral mais baixo $\mathrm{VO}_{2 \text { máx }}$ $(p=0,001)$ e obesidade central mais baixo $V_{2 \text { máx }}(p=0,024)$ apresentaram maior ocorrência de hipertensão arterial (Qui-quadrado para

Tabela 1. Variáveis gerais estratificadas por sexo.

\begin{tabular}{|c|c|c|c|}
\hline Variáveis & $\begin{array}{c}\text { Homens }(n=429) \\
\text { Média } \pm \text { DP }\end{array}$ & $\begin{array}{c}\text { Mulheres }(n=663) \\
\text { Média } \pm \text { DP }\end{array}$ & $p$ \\
\hline Idade (anos) & $27,4 \pm 10$ & $27,8 \pm 10$ & 0,602 \\
\hline $\mathrm{IMC}\left(\mathrm{kg} / \mathrm{m}^{2}\right)$ & $24,5 \pm 3,7$ & $22,1 \pm 3,4$ & 0,001 \\
\hline $\mathrm{CC}(\mathrm{cm})$ & $84,3 \pm 10,5$ & $73,4 \pm 8,2$ & 0,001 \\
\hline PAS $(\mathrm{mmHg})$ & $124,5 \pm 12,5$ & $111,8 \pm 10,1$ & 0,001 \\
\hline PAD $(\mathrm{mmHg})$ & $80,1 \pm 8,1$ & $71,5 \pm 7,6$ & 0,001 \\
\hline PAM $(\mathrm{mmHg})$ & $94,9 \pm 8,6$ & $84,9 \pm 7,9$ & 0,001 \\
\hline $\mathrm{VO}_{2 \operatorname{máx}}(\mathrm{ml} / \mathrm{kg} / \mathrm{min})$ & $34,4 \pm 10,6$ & $35,4 \pm 11,1$ & 0,177 \\
\hline
\end{tabular}

$\mathrm{DP}=$ desvio padrão; $I M C$ = índice de massa corporal; $C C$ = circunferência de cintura; $P A S=$ pressão arterial sistólica; $\mathrm{PAD}$ = pressão arterial diastólica; $\mathrm{PAM}$ = pressão arterial média; $\mathrm{VO}_{2 \mathrm{max}}=$ consumo máximo de oxigênio

Tabela 2. Associação entre hipertensão arterial e fatores de risco cardiovascular em adultos sedentários.

\begin{tabular}{c|c|c|c}
\hline \multirow{2}{*}{$\begin{array}{c}\text { Fatores de risco } \\
\text { cardiovascular }\end{array}$} & & \multicolumn{2}{|c}{ Hipertensão arterial } \\
\cline { 3 - 4 } & & $\mathbf{( n = 1 0 2 )}$ & $\mathbf{p}$ \\
\hline $\mathrm{IMC}\left(\mathrm{kg} / \mathrm{m}^{2}\right)$ & $<30 \mathrm{~kg} / \mathrm{m}^{2}$ & $7,8 \%$ & 0,001 \\
\hline $\mathrm{CC}(\mathrm{cm})$ & $<30 \mathrm{~kg} / \mathrm{m}^{2}$ & $44,7 \%$ & \\
\hline & $<102^{\text {homem } /<88^{\text {mulheres }}}$ & $8,1 \%$ & 0,001 \\
\hline $\mathrm{VO}_{2 \mathrm{máx}}(\mathrm{ml} / \mathrm{kg} / \mathrm{min})$ & $\geq 102^{\text {homem } / \geq 88^{\text {mulheres }}}$ & $30 \%$ & \\
\hline & $\geq \mathrm{P} 25$ & $8 \%$ & 0,013 \\
\hline
\end{tabular}

$\mathrm{DP}$ = desvio padrão; IMC = índice de massa corporal; $C \mathrm{CC}=$ circunferência de cintura; $P A S$ = pressão arteria sistólica; $\mathrm{PAD}=$ pressão arterial diastólica; $\mathrm{PAM}$ = pressão arterial média; $\mathrm{VO}_{2 \text { máx }}=$ consumo máximo de oxigênio

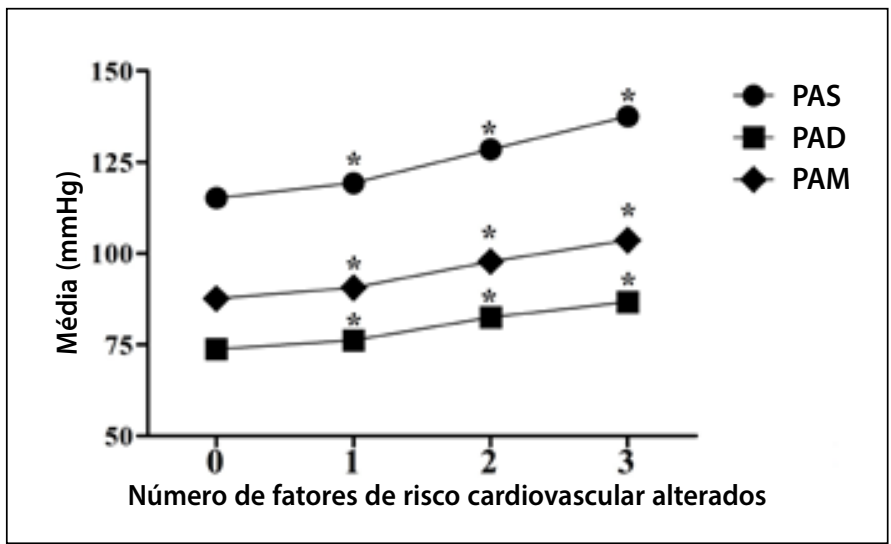

Nota. * = teste post hoc de Tukey com $p<0,05$. comparado a 0 variáveis alteradasGrupo " 1 fator de risco cardiovascular" foi composto como segue: $88,4 \%$ baixo $\mathrm{VO}_{2 \max } 8 \%$ alta CC e 3,6\% alto IMC; grupo "2 fatores de risco cardiovascular" foi composto como segue: $42,9 \%$ baixo $\mathrm{VO}_{2 \operatorname{maxx}}+$ alta $\mathrm{CC} / 38,1 \%$ baixo $\mathrm{VO}_{2 \text { máx }}+$ alto IMC / 19\% alta WC + alto IMC

Figura 1. Comparação de valores de pressão arterial de acordo com o número de fatores de risco cardiovascular entre adultos sedentários. tendência linear com $p=0,001)$. A mais alta ocorrência de hipertensão arterial foi identificada no grupo sedentário com diagnóstico positivo para todos os fatores de risco analisados (figura 2A).

Finalmente, a associação entre hipertensão arterial e agregação de fatores de risco cardiovascular foi testada em um modelo multivariado, o qual foi ajustado por sexo e idade. Independentemente do ajuste estatístico feito (sexo e idade), indivíduos sedentários com simultâneo decréscimo de aptidão cardiorrespiratório e obesidade geral $(\mathrm{RP}=5,21$ [IC95\% = 8,94 - 3,03]; $p=0,001)$ e central (RP = 4,05 [IC95\%=2,22 - 7,40]; $\mathrm{p}=0,001)$ apresentaram mais alta probabilidade de ser hipertenso do que indivíduos com nenhum fator de risco cardiovascular (figura 2B).

\section{A}

\section{Fatores de risco cardiovascular modificados}

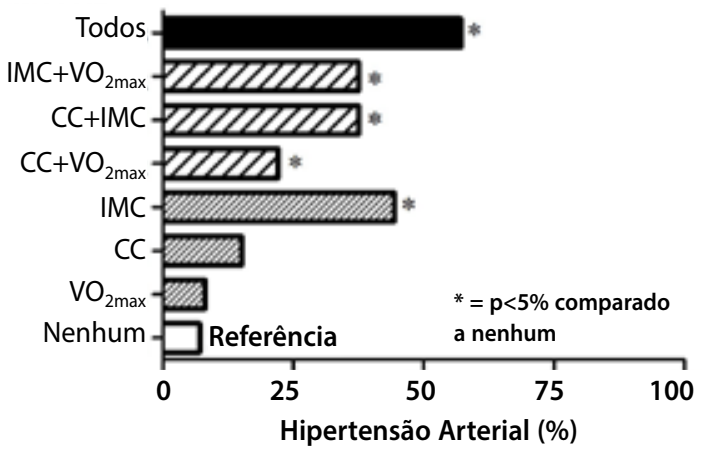

Fatores de risco cardiovascular modificados

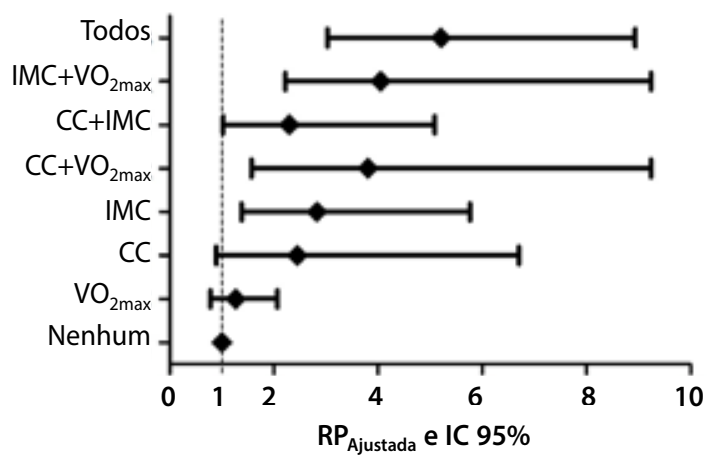

Nota. IMC = alto índice de massa corporal (obesidade, IMC $\left.\geq 30 \mathrm{~kg} / \mathrm{m}^{2}\right) ; C C=$ circunferência de cintura (obesidade abdominal, $C C \geq 88 \mathrm{~cm}$ para mulheres ou $\geq 102 \mathrm{~cm}$ para homens): $\mathrm{VO}_{2 \mathrm{ix}}=$ consumo máximo de oxigênio (baixa aptidão cardiorrespiratória, valores de $\mathrm{VO}_{2 \operatorname{máx}}$ no mais baixo quartil ajustado por sexo e idade).

Figura 2. Modelo multivariado com e sem ajuste para associação entre fatores de risco cardiovascular e pressão arterial entre adultos sedentários.

\section{DISCUSSÃO}

Hipertensão arterial está associada com disfunção endotelial, a qual é caracterizada por ausência ou mau funcionamento dos mecanismos de vasodilatação iniciados pelo tecido endotelial ${ }^{3,5}$. A obesidade é relacionada à disfunção endotelial por meio de diferentes vias, tais como produção de adipocinas e oxidação de lipoproteínas de baixa densidade por espécies reativas de oxigênio ${ }^{3,5}$. Dessa fora, a associação entre hipertensão arterial e obesidade geral/abdominal observada em nosso estudo foi esperada e corrobora com estudos prévios ${ }^{7,8}$.

Por outro lado, não é comum na literatura nacional encontrar estudos 
que discriminam a presença da obesidade abdominal em adultos com sobrepeso; da mesma forma, não é usual discriminar nas análises adultos com obesidade abdominal que têm ou não excesso de peso. Nossos achados indicam que esta discriminação é importante. Surpreendentemente, a obesidade abdominal constituiu a variável significantemente associada com a hipertensão arterial apenas se acompanhada da obesidade geral e baixa aptidão cardiorrespiratória. Aparentemente, os pontos de corte para CC (homens: $102 \mathrm{~cm}$ e mulheres: $88 \mathrm{~cm}$ ) poderiam não ser adequados para discriminar adultos brasileiros em mais alto risco para hipertensão arterial ${ }^{17}$.

Aptidão física foi inversamente relacionada com pressão arterial nesses adultos. É bem documentado que maior aptidão cardiorrespiratória protege a estrutura dos vasos sanguíneos e está associada com maior integridade do endotélio. Durante o exercício físico, o atrito causado pelo maior fluxo sanguíneo na parede dos vasos (shear stress) é responsável pela ativação de vias enzimáticas relacionadas à maior liberação de antioxidantes, os quais inativam espécies reativas de oxigênio e, consequentemente, diminuem o estresse oxidativo, aumentando a disponibilidade de óxido nítrico ${ }^{1}$. Adicionalmente, maior aptidão cardiorrespiratória está relacionada com maior produção de óxido nítrico ${ }^{18}$, aumento da atividade parassimpática ${ }^{19}$ e decréscimo da adiposidade 20

Por outro lado, baixo $\mathrm{VO}_{2 \text { máx }}$ foi associado com hipertensão arterial apenas se o indivíduo com baixo valor de $\mathrm{VO}_{2 \text { máx }}$ apresentou também simultaneamente a obesidade geral ou central. O possível mecanismo relacionado a este achado seria o efeito da aptidão cardiorrespiratória nas concentrações sanguíneas de adipocinas liberadas pelo tecido adiposo. Em pacientes diabéticos, treinando duas vezes por semana (12 meses de duração) tanto treinamento resistido (20 minutos: $80 \%$ de uma repetição máxima) quanto aeróbio (40 minutos: $70-80 \%$ do $\mathrm{VO}_{2 \text { máx }}$ ), há aumento significativo do $\mathrm{VO}_{2 \text { máx }}$ em $25,5 \%$, decréscimo nas concentrações sanguíneas de agentes pró-inflamatórios (fator de necrose tumoral alfa e interleucina-6) e aumento nas concentrações de agentes anti-inflamatórios (adiponectina) $^{21}$.

A concentração sanguínea dessas adipocinas está relacionada à resistência à insulina, a qual é fortemente relacionada ao aumento da pressão arterial, pois diminui a vasodilatação mediada pela insulina e aumenta a ativação de endotelina-1 produzida pelas células endoteliais ${ }^{4}$. Assim, mesmo em pessoas com reduzido $\mathrm{VO}_{2 \text { máx }}$ a pressão arterial será mais elevada apenas na presença da obesidade e seu status de produção aumentada de adipocinas pró-inflamatórias. 0 mecanismo hipotético acima mencionado é fortalecido pelo fato que mais alta taxa de hipertensão arterial tem sido identificada em grupos que simultaneamente apresentam baixa aptidão cardiorrespiratória, obesidade geral e total.

Em nosso estudo, o tamanho da amostra garante poder para as análises; contudo, a ausência de causalidade gerada pelo delineamento transversal precisa ser considerada como a principal limitação do estudo. Além disso, nosso estudo não considerou outros fatores de risco cardiovascular, caso do etilismo e hábitos alimentares.

\section{CONCLUSÃO}

Em resumo, nossos achados indicam que, em adultos sedentários, independentemente de idade e sexo, a presença de baixa aptidão cardiorrespiratória está associada à hipertensão arterial apenas quando também há a obesidade geral ou abdominal, indicando que a interação destas variáveis tem relevante peso na gênese da doença.

Todos os autores declararam não haver qualquer potencial conflito de interesses referente a este artigo.

\section{REFERÊNCIAS}

1. de Moraes C, Davel AP, Rossoni LV, Antunes E, Zanesco A. Exercise training improves relaxation response and SOD-1 expression in aortic and mesenteric rings from high caloric diet-fed rats. BMC Physiol 2008;8:12.

2. Pohjantähti-Maaroos H, Palomäki A, Kankkunen P, Laitinen R, Husgafvel S, Oksanen K. Circulating oxidized low density lipoproteins and arterial elasticity: comparison between men with metabolic syndrome and physically active counterparts. Cardiovasc Diabetol 2010;9:41.

3. Van Gaal LF, Mertens IL, De Block CE. Mechanisms linking obesity with cardiovascular disease. Nature 2006:444:875-80.

4. Huang PL. eNOS, metabolic syndrome and cardiovascular disease. Trends Endocrinol Metab 2009;20:295-302.

5. Kotsis V, Stabouli S, Papakatsika S, Rizos Z, Parati G. Mechanisms of obesity-induced hypertension. Hypertens Res 2010;33:386-93.

6. Ong KL, Cheung BM, Man YB, Lau CP, Lam KS. Prevalence, awareness, treatment, and control of hypertension among United States adults 1999-2004. Hypertension 2007;49:69-75.

7. Yang J, Lu F, Zhang C, Liu Z, Zhao Y, Gao F, et al. Prevalence of prehypertension and hypertension in a Chinese rural area from 1991 to 2007. Hypertens Res 2010;33:331-7.

8. Fernandes RA, Zanesco A. Early physical activity promotes lower prevalence of chronicle diseases in adulthood. Hypertens Res 2010;33:926-31.

9. Sichieri R, do Nascimento S, Coutinho W. The burden of hospitalization due to overweight and obesity in Brazil. Cad Saude Publica 2007;23:1721-7.

10. Ortega FB, Ruiz JR, Hurtig-Wennlöf A, Sjöström M. Physically active adolescents are more likely to have a healthier cardiovascular fitness level independently of their adiposity status. The European Youth Heart Study. Rev Esp Cardiol 2008;61:123-9.

11. Fernandes RA, Christofaro DG, Casonatto J, Costa Rosa CS, Costa FF, Freitas Junior IF, Luiz Monteiro H, Oliveira AR. Leisure time behaviors: prevalence, correlates and associations with overweight in Brazilian adults. A cross-sectional analysis. Rev Med Chil 2010;138:29-35.
12. Perloff D, Grim C, Flack J, Frohlich ED, Hill M, McDonald M, et al. Human blood pressure determination by sphygmomanometry. Circulation 1993;88:2460-70.

13. Gordon CC, Chumlea WC, Roche AF. Stature, recumbent length, and weight. In: Lohman TG, Roche AF, Martorell R, editors. Anthropometric standardization reference manual. Champaign: Human Kinetics Books; 1988.

14. Callaway CW, Chumlea WC, Bouchard CR, Himes JH, Lohman TG, Martin AD, et al. Circumferences In: Lohman TG, Roche AF, Martorell R, editors. Anthropometric standardization reference manual. Champaign: Human Kinetics Books; 1988

15. Lean ME, Han TS, Morrison CE. Waist circumference as a measure for indicating need for weight management. BMJ 1995;311:158-61.

16. Astrand PO, Rodahl K. Textbook of work physiology. New York: McGraw Hill; 1986.

17. Peixoto Mdo R, Benicio MH, Latorre MdoR, Jardim PC. Waist circumference and body mass index as predictors of hypertension. Arq Bras Cardiol 2006;87:462-70.

18. Zaros PR, Pires CE, Bacci M Jr, Moraes C, Zanesco A. Effect of 6-months of physical exercise on the nitrate/nitrite levels in hypertensive postmenopausal women. BMC Womens Health 2009;9:17.

19. Gutin B, Barbeau P, Litaker MS, Ferguson M, Owens S. Heart rate variability in obese children: relations to total body and visceral adiposity, and changes with physical training and detraining Obes Res 2000;8:12-9.

20. Donnelly JE, Blair SN, Jakicic JM, Manore MM, Rankin JW, Smith BK; American College of Sports Medicine. American College of Sports Medicine Position Stand. Appropriate physical activity intervention strategies for weight loss and prevention of weight regain for adults. Med Sci Sports Exerc 2009:41:459-71.

21. Balducci S, Zanuso S, Nicolucci A, Fernando F, Cavallo S, Cardelli P, et al. Anti-inflammatory effect of exercise training in subjects with type 2 diabetes and the metabolic syndrome is dependent on exercise modalities and independent of weight loss. Nutr Metab Cardiovasc Dis 2010;20:608-17. 